\title{
Tromboflebite pélvica séptica em puérpera: relato de caso
}

\section{Septic pelvic tromboflebite in puerpera: case report}

\author{
Mayanna Oliveira Rolim¹. Nadiejda Mendonça Aguiar Nobre . Marcelo Lopes Barbosaํ. Emilcy Rebouças \\ Gonçalves ${ }^{1}$. Danielle Mourão Martins ${ }^{1}$. Camila Sampaio Nogueira ${ }^{2}$. \\ 1 Maternidade Escola Assis Chateaubriand (MEAC), Universidade Federal do Ceará (UFC), Fortaleza, Ceará, Brasil. 2 Universidade \\ Federal do Ceará (UFC), Fortaleza, Ceará, Brasil.
}

\section{RESUMO}

Introdução: A trombose de veia ovariana (TVO) é uma condição rara mas potencialmente grave, ocorrendo predominantemente no período pós-parto. A clínica é inespecífica, geralmente manifestando-se como síndrome abdominal dolorosa febril. Relato de caso: Paciente do sexo feminino, 32 anos, gestante (G4P4 vaginais), a termo com quadro clínico de convulsão seguida de parada cardiorrespiratória no hospital de origem. Evoluiu para parto vaginal após drogas sedativas e hemorragia puerperal levando a choque hipovolêmico revertido após drogas vasoativas. Paciente iniciou quadro de febre persistente apesar de culturas negativas e uso de antibióticos de largo espectro. Realizado tomografia computadorizada (TC) de abdome e pelve que evidenciou tromboflebite de veia gonadal direita. Iniciado anticoagulação plena com melhora do quadro febril após 48 horas. Conclusão: A paciente do caso apresentou melhora clínica logo em seguida a anticoagulação e não houve efeitos adversos. Nos últimos 20 anos, a introdução da TC e da ressonância magnética (RNM) revolucionou o diagnóstico de tromboflebite pélvica que permite avaliar o diagnóstico e inclusive a resposta à terapia com heparina. O manejo anticoagulante da TVO persiste controverso até os dias atuais, porém quando associado à antibióticos apresentou-se segura e com boa reposta clínica.

Palavras-chave: Tromboflebite. Ovário. Febre. Puerpério. Pós-Parto. Anticoagulantes.

\section{ABSTRACT}

Introduction: Ovarian vein thrombosis (OVT) is a rare, but potentially grave condition, occurring predominantly during postpartum period. The clinical presentation is non-specific, usually expressed as a feverish painful abdominal syndrome. Case report: A female patient, 32 years old, pregnant (P4,B4 vaginal), term, with clinical picture of convulsion followed by cardiopulmonary arrest in the hospital of origin. Evolved to vaginal delivery after sedative drugs and puerperal bleeding entailing to hypovolemic shock, reversed after vasoactive drugs. Patient started persistent fever picture despite negative culture and use of wide-spectrum antibiotics. It was performed a computed tomography of abdomen and pelvis that highlighted thrombophlebitis of the right gonadal vein. It was started full anticoagulation with recovery of the fever after 48 hours. Conclusion: The patient presented clinical recovery right after the anticoagulation and there were no adverse effects. In the last 20 years, the introduction of computed tomography and magnetic resonance revolutionized the diagnosis of pelvic thrombophlebitis, which allowed to evaluate the diagnosis and moreover the response to the therapy with heparin. The anticoagulant management of ovarian vein thrombosis persists controversial until the current days, however when associated to antibiotics presented secure and good clinical response.

Keywords: Thrombophlebitis. Ovary. Fever. Puerperium. Postpartum. Anticoagulants.

Autor correspondente: Mayanna Oliveira Rolim, Rua Juazeiro do Norte, número 199, Meireles, Fortaleza, Ceará. CEP: 60165-110. Telefone: +55 85 98896-1890. E-mail: mayannarolim@hotmail.com

Conflito de interesses: Não há qualquer conflito de interesses por parte de qualquer um dos autores.

Recebido em: 15 Ago 2018; Revisado em: 08 Dez 2018; Aceito em: 08 Dez 2018. 


\section{INTRODUÇÃO}

A trombose de veia ovariana (TVO) é uma condição rara, mas potencialmente grave e que pode estar associada mais comumente ao puerpério, abortamentos, doença inflamatória pélvica, cirurgias pélvicas e malignidades. ${ }^{1,2}$

A sua incidência é estimada em 0,05-0,18\%. É diagnosticado com mais frequência no lado direito dos pacientes, em até $80-90 \%$ dos casos.

A fisiopatologia da TVO relaciona-se à tríade de Virchow: lesão endotelial, estase venosa e hipercoagulabilidade. A estase venosa e a hipercoagulabilidade geralmente acompanham o puerpério; e a lesão sobreposta à parede da veia geralmente é causada por infecção uterina. ${ }^{2}$ A patogênese inclui danos à camada íntima da veia pélvica causada por uma infecção uterina disseminada, bacteremia e endotoxinas, que também podem ocorrer secundárias ao trauma do delivramento vaginal ou pós-cirúrgica. ${ }^{4}$

A clínica é bastante inespecífica, geralmente manifestando-se como síndrome que cursa com dor abdominal e febre. Pode simular apendicite, abscesso tubo-ovariano, pielonefrite e hematomas intrabdominais. O diagnóstico é desafiador e ainda é por exclusão. ${ }^{1,5}$

Os exames de imagem são fundamentais para elucidação diagnóstica, incluindo ultrassonografia com doppler, TC e RNM. É típica a persistência do quadro febril apesar de antibioticoterapia e a melhora clínica após uma média de 48 horas de anticoagulação associada. ${ }^{2}$

Relatamos um caso de tromboflebite pós-parto em veia ovariana apresentando-se com febre refratária ao tratamento com antimicrobianos e culturas negativas.

\section{RELATO DE CASO}

F. M. S, 32 anos, G4P4vA0, procedente de Paracuru-CE; chegou à emergência da Maternidade Escola Assis Chateaubriand (MEAC-UFC) em 23/07/15 com 40 semanas e 2 dias de gestação com relato de convulsão seguida de parada cardiorrespiratória (revertida após 3 ciclos de reanimação). O fato ocorreu após indução de trabalho de parto com Misoprostol (2 doses). A paciente foi admitida na emergência da MEAC hipotensa, bradicárdica, taquipneica, ECG 9T e batimentos cardiofetais presentes. Prontamente ela foi encaminhada à UTI e evoluiu para parto vaginal 1 hora após drogas sedativas. O parto foi realizado sem intercorrências, evoluindo, posteriormente, com hemorragia puerperal necessitando de drogas vasoativas, transfusão de sangue e plaquetas. Foi realizado uma curagem com evidência de restos ovulares intra-útero. Durante a permanência na UTI, a paciente apresentou-se instável e foram levantadas hipóteses diagnósticas de Eclâmpsia ou Embolia amniótica associado a Síndrome HELLP completa, seguido de choque hemorrágico (hemorragia puerperal). No primeiro dia pósparto, observou-se febre e tosse produtiva sendo iniciado piperacilina/ tazobactam (TAZOCIN). A paciente evolui no $3^{\circ}$ dia pós-parto com desconforto respiratório, ausculta pulmonar diminuída em hemitórax direito com creptações bibasais, Raio-x de tórax com aumento da área cardíaca e inversão da trama vascular e ecocardiograma normal. Mantevese com queixa de febre persistente, cefaleia, dor pélvica intensa e adinamia. Ausência de rigidez de nuca e outras alterações neurológicas ou pulmonares. A ferida operatória apresentava-se sem sinais flogísticos. Tendo em vista a febre a esclarecer, foi modificado antibiótico para meropenem e associado vancomicina e polimixina B ao esquema antimicrobiano. Urinocultura e hemoculturas apresentavam-se normais. A paciente permaneceu na UTI por febre persistente a esclarecer. Foi realizado TC de crânio normal. A tomografia de abdome e pelve evidenciou tromboflebite de veia gonadal direita (Figuras 1, 2 e 3) com líquido em fossa ilíaca direita; derrame pleural bilateral com atelectasia de restrição. Foi iniciado anticoagulação plena e a paciente evoluiu afebril após 48 horas. Ao todo, foram 21 dias de internação na UTI por Eclâmpsia, Síndrome HELLP, choque hipovolêmico e tromboflebite pélvica séptica.

Figura 1. TC corte axial fase portal apresenta veia gonadal direita difusamente aumentado calibre com material hipoatenuante no interior sem evidência de realce. Associa-se a identificação dos planos adiposos adjacentes sugerindo componente inflamatório, conjunto dos achados compatível com tromboflebite de toda extensão de veia gonadal direita. Observa-se útero gravídico a central da imagem.

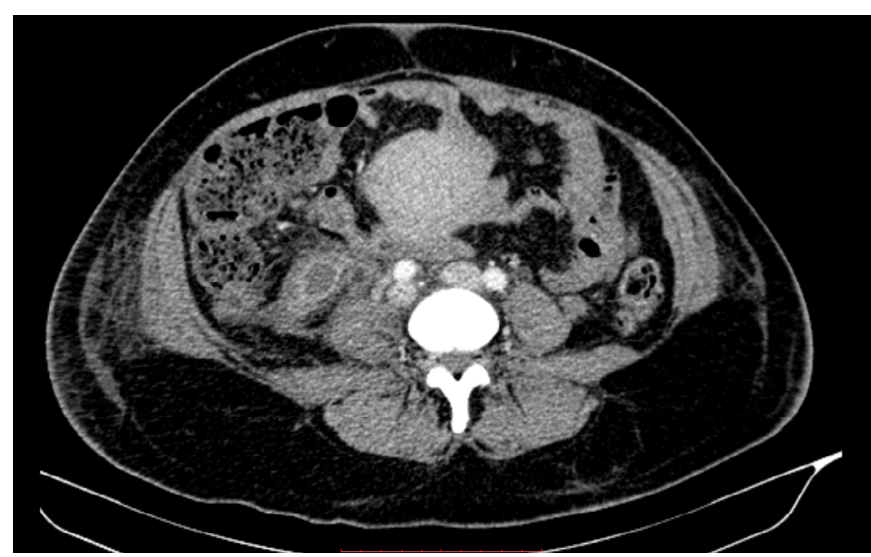

Figura 2. TC corte axial.

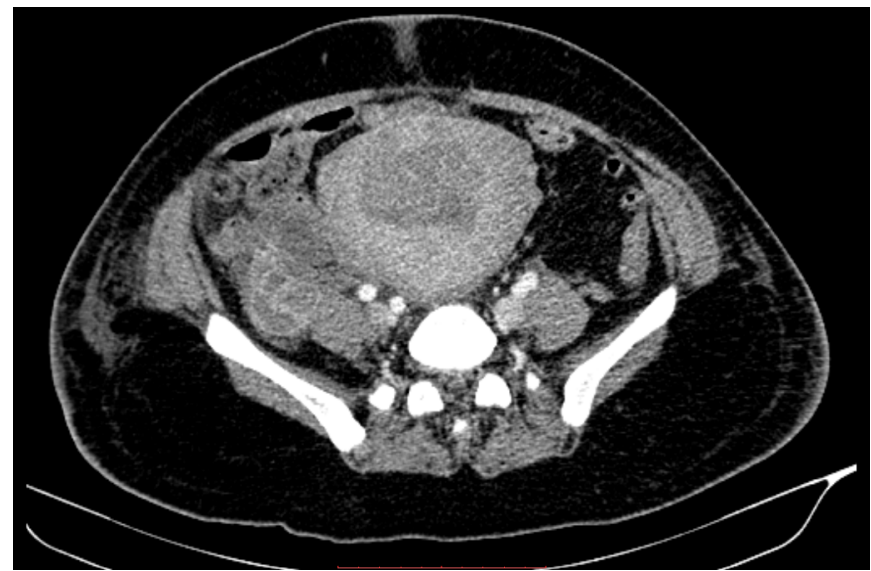


Figura 3. TC corte coronal.

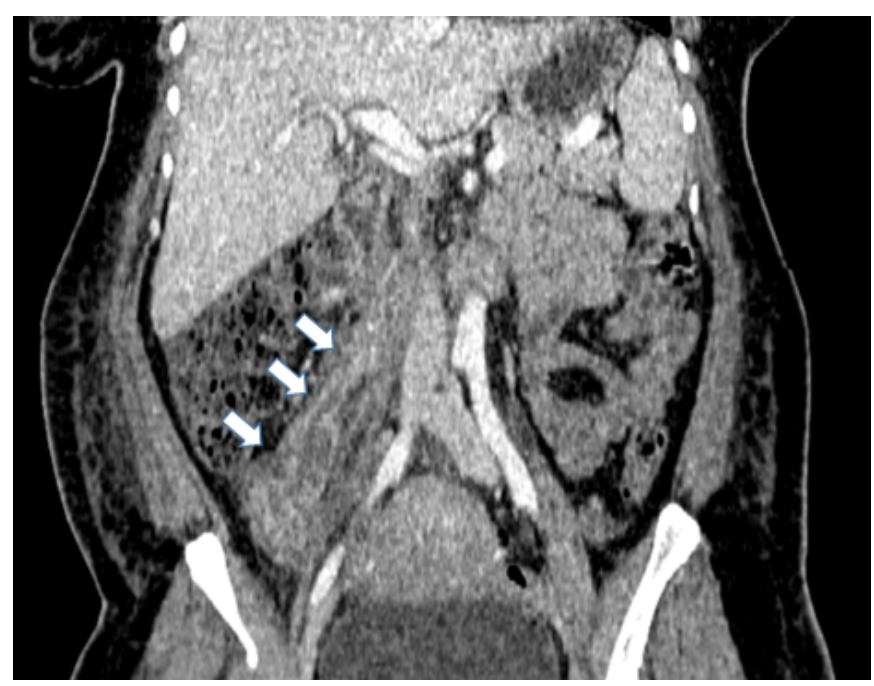

\section{DISCUSSÃO}

As infecções das paredes vasculares são usualmente associadas com bacteremia e morbimortalidade elevadas. Três vias explicam o aparecimento dos quadros de tromboflebite séptica:

1. Canulação endovascular prolongada, em geral com duração superior a $72 \mathrm{~h}$ (componente endovascular), principalmente quando são utilizadas cânulas de material plástico do tipo polietileno ao invés de teflon ou silastic;

2. Implante hematogênico a partir de um foco infeccioso distante;

3. Inflamação adjacente à parede vascular com migração de microorganismos através da rede linfática ou pelo suprimento vascular da própria parede da veia resultando assim em supuração focal local (componente perivascular). ${ }^{6}$

Em 1956, Austin escreveu pela primeira vez uma trombose de veia ovariana (TVO). Embora seja uma complicação rara, ocorre predominantemente durante o período pós-parto. Rottenstreich et $\mathrm{al}^{7}$ observaram $80 \%$ dos casos relacionados à gestação, predominantemente na fase do puerpério. Também está associado a outras causas, tais como cirurgia pélvica prévia, malignidade, doença inflamatória intestinal e doença inflamatória pélvica. ${ }^{6}$

A trombose da veia ovariana (TVO) puerperal é uma doença incomum, mas importante, porque tem evolução severa e pode resultar em uma condição fatal, como os êmbolos pulmonares sépticos. Os sintomas de febre e dor abdominal são inespecíficos e dificultam o diagnóstico clínico. A febre é prolongada apesar de antibioticoterapia apropriada, associada a dor abdominal inferior e flanco. A dor pode ser de intensidade variável, constante e irradiar para a virilha ou abdome superior. Outras queixas inespecíficas, como náuseas ou astenia, também podem ocorrer.
Durante a gravidez, há uma triplicação do diâmetro da veia ovariana e aumento de 60 vezes no volume sanguíneo da veia ovariana, o que precipita a incompetência valvar ovariana. ${ }^{8}$ Imediatamente após o parto, há uma estagnação repentina do sangue nas veias dilatadas, especialmente por trás dos folhetos da válvula. Isso, combinado com a contração do volume, lesão intimal por parto ou cesariana, sepse, estase e hipercoagulabilidade relacionada à gravidez, cumprem as condições necessárias para a formação de trombo. ${ }^{9}$

A TVO pode ser unilateral ou bilateral sendo a veia ovariana direita afetada cinco vezes mais do que a veia ovariana esquerda ( $80 \%$ e $90 \%$ casos). Há uma compressão da veia ovariana direita por dextroversão do útero gravídico (posição mais frequente), drenagem retrógrada das veias ovarianas e uterinas esquerdas na veia ovariana direita, com fluxo anterógrado persistente na veia direita após o parto. Tudo isso resulta em maior estase e inóculo bacteriano à direita. Espera-se que o curso mais longo e as múltiplas válvulas à direita sejam fatores contribuintes adicionais, enquanto que uma tendência para o fluxo retrógrado na veia ovariana esquerda geralmente o poupa. ${ }^{10,11}$

A paciente do caso permaneceu com quadro febril apesar de completa investigação para foco pulmonar, trato urinário e abdominal. Foram escalonados vários antibióticos na tentativa de cobrir bactérias gram negativo, gram positivo, anaeróbicos e fungos, porém sem melhoras. Em vista da febre persistente com culturas negativas e pobre resposta com antibióticos de largo espectro, foi solicitado TC de abdome e pelve que detectou lesão sugestiva de abscesso ou trombo em veia gonadal direita. Foi iniciado heparina em dose plena, de forma empírica, apostando na hipótese de tromboflebite pélvica séptica e obteve-se resolução do quadro febril em 48 horas.

No final dos anos sessenta, a anticoagulação com heparina associado a antibióticos para tratamento da TVO ganhou aceitação geral e mostrou-se segura. Esses relatórios foram apenas observacionais. Nos últimos 20 anos, a introdução da tomografia computadorizada e a ressonância magnética revolucionou o diagnóstico de tromboflebite pélvica, o que permite aos pesquisadores avaliar o diagnóstico e inclusive a resposta à terapia com heparina. ${ }^{12}$ No entanto, o manejo anticoagulante da TVO persiste controverso.

Brown et $\mathrm{al}^{13}$ em seu estudo randomizado com 14 puérperas que apresentaram febre prolongada e TC compatível com trombose de veia ovariana não observou diferenças significativas entre os grupos que usaram apenas antibioticoterapia e antibioticoterapia+anticoagulação com heparina, em relação ao tempo de defervescência ou evolução do quadro. E concluiu, portanto, que não há indicação de uso de anticoagulantes de forma rotineira em mulheres com febre persistente pós-parto. No entanto, diversos estudos recentes ${ }^{7,12,14}$ sugerem que a anticoagulação pode ser feita por até 3 meses ou mais, dependendo do acometimento.

Falagas, ${ }^{15}$ em uma revisão sistemática, observou que em vários estudos a heparina foi adicionada ao regime terapêutico. Os antibióticos mais comumente usados foram 
cefalotina, oxacilina, ampicilina/gentamicina/clindamicina e vancomicina. Ticarcilina/clavulanato, ceftriaxona e vários aminoglicosídeos também foram utilizados. Apenas 2 pacientes (dos 115 pacientes avaliados para mortalidade) morreram após o tratamento com heparina para tromboflebite séptica. Um morreu devido ao choque séptico e outro por infecção polimicrobiana e necrose tubular renal.

A adição de heparina ao regime de tratamento não foi uniforme em todos os estudos e persistiu controverso, sendo adicionada imediatamente após a apresentação de febre, forte suspeita clínica ou tromboflebite séptica comprovada, ou alguns dias depois, quando a terapia com antibióticos foi considerada como falha. O tempo de defervescência após a introdução

\section{REFERÊNCIAS}

1. Roepke RM, Campos FP, Lovisolo SM, Santos EH. Septic pelvic thrombophlebitis of unknown origin. Autops Case Rep. 2014;4(3):39-46.

2. Khlifi A, Kebaili S, Hammami M, Berregaya L, Hidar S, Affes N, et al. Postpartum ovarian vein thrombophlebitis: report of a case and review of the literature. N Am J Med Sci. 2010;2(8):389-91.

3. Hafsa C, Golli M, Jerbi-Omezzine S, Salem R, Kriaa S, Zbidi $\mathrm{M}$, et al. A rare aetiology of the post-partum fever: ovarian vein thrombophlebitis. Ann Fr Anesth Reanim. 2006;25(3):286-90.

4. Dunnihoo DR, Gallaspy JW, Wise RB, Otterson WN. Postpartum ovarian vein thrombophlebitis: a review. Obstet Gynecol Surv. 1991;46(7):415-27.

5. Nezhat C, Farhady P, Lemyre M. Septic pelvic thrombophlebitis following laparoscopic hysterectomy. JSLS. 2009;13(1):84-6.

6. Gakhal MS, Levy HM, Spina M, Wrigley C. Ovarian vein thrombosis: analysis of patient age, etiology, and side of involvement. Del Med J.2013;85(2):45-50.

7. Rottenstreich A, Da'as N, Kleinstern G, Spectre G, Amsalem H, Kalish Y. Pregnancy and non-pregnancy related ovarian vein thrombosis: clinical course and outcome. Thromb Res. 2016;146:84-8.

8. Hodkinson CP. Physiology of the ovarian veins during pregnancy. Obstet Gynecol. 1953;1(1):26-37. da heparina variou entre os estudos e nenhuma estimativa conclusiva sobre o tempo de defervescência após a introdução da heparina pode ser extraída dos estudos revisados. No entanto, a ampla revisão sugere ser uma estratégia útil ao tratamento da tromboflebite séptica apesar de reconhecer que há pacientes onde não há benefício ou modificação com uso da heparina.

A paciente do caso relatado apresentou significativa melhora clínica após a associação da anticoagulação ao esquema antibiótico e seguiu-se de defervescência febril precoce, além de não apresentar efeitos adversos, sendo esta a conduta atualmente adotada em nosso serviço (MEAC-UFC) baseando-se nos resultados observados na literatura.

9. Kominiarek MA, Hibbard JU. Postpartum ovarian vein thrombosis: an update. Obstet Gynecol Surv. 2006;61(5):337-42.

10. Virmani V, Kaza R, Sadaf A, Fasih N, Fraser-Hill M. Ultrasound, computed tomography, and magnetic resonance imaging of ovarian vein thrombosis in obstetrical and nonobstetrical patients. Can Assoc Radiol J. 2012;63(2):109-18.

11. Johnson A, Wietfeldt ED, Dhevan V, Hassan I. Right lower quadrant pain and postpartum ovarian vein thrombosis. Uncommon but not forgotten. Arch Gynecol Obstet. 2010;281(2):261-3.

12. Garcia J, Aboujaoude R, Apuzzio J, Alvarez JR. Septic pelvic thrombophlebitis: diagnosis and management. Infect Dis Obstet Gynecol. 2006;2006:1-4.

13. Brown CE, Stettler RW, Twickler D, Cunningham FG. Puerperal septic pelvic thrombophlebitis: incidence and response to heparin therapy. Am J Obstet Gynecol. 1999;181(1):143-8.

14. Sharma P, Abdi S. Ovarian vein thrombosis. Clinical Radiology. 2012;67(9):893-8.

15. Falagas ME, Vardakas KZ, Athanasiou S. Intravenous heparin in combination with antibiotics for the treatment of deep vein septic thrombophlebitis: a systematic review. Eur J Pharmacol. 2007;557(23):93-8.

\section{Como citar:}

Rolim MO, Nobre NM, Barbosa ML, Gonçalves ER, Martins DM, Nogueira CS. Tromboflebite pélvica séptica em puérpera: relato de caso. Rev Med UFC. 2019 out-dez;59(4):70-73. 